\title{
LGR5, HES1 and ATOH1 in Young Rectal Cancer Patients in Egyptian
}

\author{
Heba Morsy ${ }^{1}$, Ahmed Gaballah ${ }^{2}$, Mohamed Samir ${ }^{3}$, Vandrome Nakundi ${ }^{4}$, \\ Mohamed Shamseya ${ }^{5}$, Hanan Mahrous ${ }^{1}$, Abeer Ghazal ${ }^{2}$, Mervat Hashish ${ }^{1}$, \\ Waleed Arafat ${ }^{4,6 *}$
}

\begin{abstract}
Objective: The study aimed to delineate the gene expression profile of LGR5, HES1 and ATOH1 in young Egyptian rectal cancer (RC) patients and investigate the correlation between expression profiles and clinical outcome. Methods: The study was conducted on 30 young Egyptian RC patients. Expression study of LGR5, HES1 and ATOH1 were performed by quantitative PCR (QPCR) based on comparative Cq method after normalization to adjacent non tumor tissues and ACTB as a reference gene. Patients were followed up for assessment of response to neoadjuvant chemoradiotherapy (CRT) based on revised RECIST1.1. Result: The study detected overexpression of LGR5 and HES1 and down-regulation of ATOH1 in human RC tissues compared to non- tumor tissues. High expression of $L G R 5$ was correlated with more depth of tumor invasion, lymph node (LN) metastasis, advanced cTNM stage and mesorectal fascia (MRF) involvement. More prominently, high LGR5 expression level was associated with poor response to CRT. LGR5 was suggested as unfavorable prognostic biomarker for RC. Conversely, HES1 and ATOH1 expression did not show significant association with most of the studied clinical criteria nor response to CRT. Still, HES1 and ATOH1 were significantly and inversely associated with presence of mucinous component. Conclusion: High LGR5 expression is indicative of poor prognosis among young Egyptian RC patients and is proposed as a predictive marker of resistance to neoadjuvant CRT. However, HES1 and ATOH1 expressions were not prognostic nor predictive of response to CRT. Overall, LGR5, HES1 and ATOH1 gene expression patterns among young onset RC patients, are in line with patterns encountered in older age groups.
\end{abstract}

Keywords: Rectal cancer- Egypt- Young adults- Cancer stem cells- Gene expression- LGR5- HES1-ATOH1

Asian Pac J Cancer Prev, 22 (9), 2819-2830

\section{Introduction}

In Egypt, Colorectal cancer (CRC) is the sixth most common cause of cancer-related deaths, with a relative frequency of $4.2 \%$ (Ibrahim et al.,2014). The percentage of young-onset CRC cases in Egyptians is strikingly high with more than one third of cases occurring under 40 years (Soliman et al., 1997; Soliman et al., 1999). Different molecular pathology and unique pathogenesis features of CRC in Egyptian compared to Western patients, was reported (Soliman et al., 2001). Since, more than half of the Egyptian population is younger than 50 years, a mounting public health problem of CRC among young adults in Egypt should be highly considered (Silla et al., 2014).

Despite extensive CRC-focused research over the past 20 years, the clinical outcome of CRC is still very poor (Siegel et al., 2015). A growing number of research proposed cancer stem cells (CSCs), as the main element contributing to tumor progression, relapse, metastasis and therapeutic resistance (Visvader et al., 2008). A thorough understanding of the specific biomarkers and the signaling pathways governing the functions of CSCs is crucial for identification of valuable prognostic markers and therapeutic targets for CRC (Chen et al., 2013).

LGR5 (Figure 1), also known as GPR49, was proposed as the most selective and promising marker of CSCs in CRC (Wu et al., 2012; Barker et al., 2014). Some studies, used the Immunohistochemistry technology to detect its expression, have shown the correlation between the LGR5 upregulation and the histopathological characteristics of CRC. Furthermore, the same results have been addressed by meta-analysis studies (Chen et al., 2014; Han et al.,

${ }^{1}$ Department of Human Genetics, Medical Research Institute, Alexandria, Egypt. ${ }^{2}$ Department of Microbiology, Medical Research Institute, Alexandria, Egypt. ${ }^{3}$ Department of Clinical and Experimental Surgery, Medical Research Institute, Alexandria, Egypt. ${ }^{4}$ Department of Clinical Oncology and Nuclear Medicine, Faculty of Medicine, Alexandria University, Egypt. ${ }^{5}$ Department of Clinical and Experimental Internal Medicine, Medical Research Institute, Alexandria, Egypt. ${ }^{6}$ Comprehensive Cancer Center of Alexandria (CCC Alex), Alexandria,EEypt.*For Correspondence: waleed_o_arafat@yahoo.com 
2015; Jiang et al., 2016). However, these studies are limited by the fact that the presence of antibodies targeting the LGR5 remains in doubt (Barker et al., 2014; Kemper et al., 2012; Baker et al.,2015).

The studies, detected the LGR5 using in situ hybridization in $\mathrm{CRC}$, have found different results; same of them have shown correlation between LGR5 upregulation and poor prognosis (Baker et al.,2015; Uchida et al., 2010) while others did not find any association (Ziskin et al., 2013; Jang et al., 2018). Thus, further research, using alternative method, is required as most previous studies have been carried out in elderly patients.

Tumors consist of distinct CSC populations with different biomarkers that the association with the histopathological features of CRC has still needed to be assessed (Nimmakayala et al., 2019). The upregulation of CD133 has been associated with metastasis and distant recurrence of CRC after chemoradiotherapy (Yasuda et al., 2009; Horst et al., 2009; Saigusa et al., 2010). The CD24 has shown controversial result, its cytoplasmic expression demonstrated correlation with invasion and differentiation but not association with reduced patient overall survival (Choi et al., 2009; Ahmed et al., 2009).

Notch signaling (Figure 2) is implicated in maintenance of CSCs and its aberrant activation plays a key role in carcinogenesis and progression of human malignancy. Several studies have revealed how Notch signaling pathway might regulate HES1 and ATOH1 expressions in normal and cancerous tissues; moreover it worth mentioning that same studies have shown that the inhibition of Notch signaling reduces the LGR5 intestinal stem cell population (Srinivasan et al., 2016).

The CSCs hypothesis raises questions regarding current diagnostic and therapeutic modalities (Vaiopoulos et al., 2012). In spite of remarkable and non-stop advances in studying CSCs in CRC, further molecular characterization is crucial especially in Egypt, where molecular aspects of CSCs have not been fully investigated among Egyptian patients.

The aim of the current study was to delineate the gene expression profile of three CSCs related genes, LGR5, HES1 and ATOH1, among young Egyptian rectal cancer patients, and investigate possible association between these genes' expression and clinical outcome, including response to neoadjuvant chemoradiotherapy.

\section{Materials and Methods}

\section{Study Participants}

The study was conducted on 30 young Egyptian RC patients (less than 40 years old) with histopathologically confirmed adenocarcinoma. Patients were recruited from Gastroenterology and Endoscopy Unit at Alexandria Main University Hospital and Medical Research Institute within the period from August 2013 to February 2015. The study was conducted in accordance with the guidelines of the Declaration of Helsinki and all participants signed an informed consent before participation in the study.

\section{Methods}

All patients enrolled in the study were thoroughly examined. Paired tumor and non-tumor adjacent mucosal tissues were obtained from patients by routine biopsy techniques during colonoscopy followed by histopathological examination. C.T abdomen \& pelvis, pelvic MRI were requested for adequate clinical staging of RC according to TNM staging, $7^{\text {th }}$ edition. Patients received neoadjuvant $\mathrm{CRT}$ comprising $3 \mathrm{D}$ conformational radiotherapy using $\mathrm{CT}$ planning to deliver a dose of 45-50.4 Gray with conventional fractionation every day except Thursday and Friday for 5-7 weeks, concurrent with daily Capecitabine $\left(825 \mathrm{~g} / \mathrm{m}^{2}\right.$ Bid from D1- D5/ week).

Evaluation of the change in tumor burden is considered a valuable feature of the clinical assessment of cancer therapeutics. As a consequence, the revised RECIST 1.1 guidelines were adopted in the current study as a primary response endpoint to neoadjuvant CRT (Eisenhauer et al., 2009). Patients were followed up and reassessed for response to neoadjuvant therapy after 6 weeks, then categorized according to RECIST guidelines 1.1 into 4 groups; complete response (CR), partial response (PR), stable disease (SD) and progressive disease (PD).

\section{Gene expression study by $q P C R$}

Paired tumor and non-tumor adjacent tissue samples were immediately fixed in RNA later stabilization reagent. Total RNA isolation from all tissue samples was carried out using RNeasy Protect Mini Kit (Qiagen, Germany) according to the manufacturer's instructions. The concentration of RNA was determined by measuring the absorbance at $260 \mathrm{~nm}\left(\mathrm{~A}_{260}\right)$ by Nanodrop (Thermo Scientific, USA). Reverse transcription of RNA to cDNA was done using TaqMan Reverse Transcription Reagents (Invitrogen, USA) according to manufacturer's protocol. PCR products were amplified from cDNA samples using the SYBR Green Universal Master Mix (Life technologies, USA), in addition to specific primers for target genes LGR5, HES1, ATOH1 according to previously published sequences (Kobayashi et al., 2012; Chen et al., 2010; Kong et al., 2012). QPCR reactions were done in triplicate for all samples and performed in Step one real time PCR (Applied Biosystems). Relative expression of target genes was calculated using the comparative $\mathrm{Cq}$ method $\left(2^{-\Delta \Delta \mathrm{Cq}}\right)$ by normalization to ACTB as a reference gene and adjacent non- tumor tissue as calibrators (Livak et al., 2001).

\section{Statistical Analysis}

Statistical analysis was done using IBM SPSS statistics program version 21 and Medcalc program. Mann-Whitney test (MW) was used to detect significant difference in the median quantitative variables between two groups of patients. Kruskal Wallis test (KW) was done to compare the median quantitative variables between the several categories of depth of tumor invasion. For significant results, pair wise comparison was done using adjusted $\mathrm{p}$ value by Bonferroni correction.

Receiver operating characteristic (ROC) curve analysis was done to detect the diagnostic accuracy of different indices for response to treatment. Area under the curve (AUC), sensitivity, specificity, positive predictive value and negative predictive value were used to evaluate each index (Leeflang et al., 2013). All statistical tests were done 
at 0.05 significance level.

\section{Results}

The present study was carried out on 30 young Egyptian patients with RC adenocarcinoma who were eligible for neoadjuvant therapy. Demographic and clinical criteria of the studied patients in addition to rectal tumor characteristics, including response to neoadjuvant CRT, are summarized in Table 1.

Relative quantitative expression of LGR5, HES1 and $A T O H 1$ in rectal tumor tissues compared to paired non-tumor adjacent tissues revealed significant overexpression of LGR5 and HES1 ( $\mathrm{p}<0.001)$. Conversely, $A T O H 1$ was significantly down regulated in tumor tissues relative to non- tumor adjacent tissues $(\mathrm{p}=0.05)$ (Figure 3).

The median LGR5 fold change was significantly correlated to MRF involvement among the studied rectal tumors $(\mathrm{p}<0.001)$ (Figure 4). A substantially higher levels of $L G R 5$ expression were noted in tumors with involved MRF compared to those with free MRF. Likewise, statistically significant difference was detected in median $L G R 5$ expression level along different staging parameters. Particularly, LGR5 expression levels significantly correlated with depth of tumor invasion $(p=0.002)$. Post hoc paired comparisons revealed that median expression levels of LGR5 in cT4 was significantly higher than in $\mathrm{cT} 1 / 2$ (adjusted $\mathrm{p}=0.002$ ) and $\mathrm{cT} 3$ (adjusted $\mathrm{p}=0.031$ ). However, $L G R 5$ expression levels didn`t show significant difference between cT1/2 and cT3 (adjusted $\mathrm{p}=0.520$ ). Furthermore, Expression of $L G R 5$ was significantly higher in patients with regional LN metastasis compared to patients without LN metastasis $(\mathrm{p}<0.001)$. Consequently, stage III rectal tumors showed highly significant overexpression of LGR5 compared to stage II. (p $<0.001)$. On the contrary, no significant correlations were observed between relative $L G R 5$ expression and patients demographic characteristics (age and sex), tumor location nor pathological features (Table 2, Figure 3).

HES1 expression levels in distal rectal tumors ranged from 0.59 to 42.67 with a median of 8.28 , while in proximal rectal tumors it ranged from 0.6 to 62.05 with a median of 3.39. Hence, tumors in Lower $2 / 3$ of rectum had significantly higher HES1 expression levels than those in Upper $1 / 3(p=0.019)$. Statistical analysis revealed no significant correlation between HES1 relative expression and pathological grade of differentiation $(\mathrm{p}=0.402)$. Still, a statistically significant association was identified between HES1 relative expression levels and presence of mucinous component in the studied rectal adenocarcinomas $(p=0.022)$. The median HES1 fold change in rectal tumors with mucinous components was significantly lower than non- mucinous tumors. On the other hand, no significant difference was observed in HES1 expression levels as regards MRF involvement $(p=0.28)$ and different staging parameters, including $\mathrm{cT}$ $(\mathrm{p}=0.749), \mathrm{cN}(\mathrm{p}=0.103)$ and $\mathrm{cTNM}$ staging $(\mathrm{p}=0.233)$. Additionally, median HES1 fold change didn`t show statistically significant difference with RC patients' demographic characteristics (Age and sex) (Table 2).
LGR5, HES1 and ATOH1 in Young Rectal Cancer Egyptian Patients

ATOH1 median fold change was significantly lower among RC patients under 30 years old compared to older patients aged $30-40$ years $(\mathrm{p}=0.037)$. Though, no significant difference in expression levels of $A T O H 1$ was

Table 1. Demographic and Clinical Characteristics of RC Patients

\begin{tabular}{lc}
\hline Characteristics & No. $(\%)$ \\
\hline Sex & $17(56.7)$ \\
$\quad$ Male & $13(43.3)$ \\
Female & \\
Age & $11(36.7)$ \\
20- 30 years & $19(63.3)$ \\
30-40 years & \\
Clinical presentation & $21(70)$ \\
Bleeding per rectum & $13(43.3)$ \\
Pain and tenesmus & $12(40)$ \\
Constipation & $4(13.3)$ \\
Mucous in stool & \\
Rectal tumor site & $12(40)$ \\
Upper $1 / 3$ & $18(60)$ \\
Lower $2 / 3$ & \\
Tumor grade & $6(20)$ \\
Low grade & $24(80)$ \\
High grade &
\end{tabular}

Adenocarcinoma with mucinous component

Present $7(23.3)$

Absent $23(76.6)$

MRF involvement

Positive

Negative

$17(56.7)$

Clinical staging

cT

$\mathrm{cT} 1 / 2$

cT3 $14(46.6)$

cT4 $8(26.7)$

$\mathrm{cN}$

cN0 $13(43.3)$

cN1 $11(36.7)$

cN2 $6(20)$

$\mathrm{cM}$

cM0 $30(100)$

cTNM

$\begin{array}{ll}\text { stage II } & 15(50) \\ \text { stage III } & 15(50)\end{array}$

Response to neoadjuvant CRT based on RECIST 1.1 guidelines

$\begin{array}{lc}\text { CR } & 8(26.7) \\ \text { PR } & 6(20) \\ \text { SD } & 6(20) \\ \text { PD } & 10(33.3)\end{array}$

MRF, Mesorectal fascia; CR, complete response; PR, Partial response; $\mathrm{SD}$, Stable disease; PD, Progressive disease

Asian Pacific Journal of Cancer Prevention, Vol 22 
Table 2. Correlation between LGR5, HES1\& ATOH1 Genes Fold Change and Clinicopathological Criteria of the Studied RC Patients

\begin{tabular}{|c|c|c|c|c|c|c|c|}
\hline \multicolumn{8}{|c|}{$\begin{array}{l}\text { Clinicopathological } \\
\text { criteria }\end{array}$} \\
\hline & & $\begin{array}{l}\text { LGR5 fold } \\
\text { change }\end{array}$ & Statist. sig. & $\begin{array}{l}\text { HES1 fold } \\
\text { change }\end{array}$ & Statist. sig. & $\begin{array}{l}\text { ATOH1 fold } \\
\text { change }\end{array}$ & Statist. sig. \\
\hline & & Median (min-max) & & Median (min-max) & & Median (min-max) & \\
\hline \multirow[t]{2}{*}{ Age } & $<30 y$ & $53.73(2.36-382.06)$ & ${ }^{\mathrm{MW}} \mathrm{p}=0.471$ & $4.45(0.59-42.67)$ & ${ }^{\mathrm{MW}} \mathrm{p}=0.35$ & $0.17(0.002-1.68)$ & ${ }^{\mathrm{MW}} \mathrm{p}=0.037^{*}$ \\
\hline & $30-40 y$ & $27.81(4.11-426.87)$ & & $5.59(0.6-62.05)$ & & $0.74(0.02-3.86)$ & \\
\hline \multirow[t]{2}{*}{$\operatorname{Sex}$} & Male & $30.65(2.36-382.06)$ & ${ }^{\mathrm{MW}} \mathrm{p}=0.536$ & $5.59(0.59-62.05)$ & ${ }^{\mathrm{MW}} \mathrm{p}=1.00$ & $0.21(0.00-3.86)$ & ${ }^{\mathrm{Mw}} \mathrm{p}=0.51$ \\
\hline & Female & $34.7188(11.22-426.87)$ & & $5.41(1.44-36.64)$ & & $0.38(0.10-2.03)$ & \\
\hline \multirow{2}{*}{$\begin{array}{l}\text { Tumor } \\
\text { location }\end{array}$} & U $1 / 3$ & $36.84(2.41-426.87)$ & ${ }^{\mathrm{MW}} \mathrm{p}=0.723$ & $3.39(0.60-62.05)$ & ${ }^{\mathrm{MW}} \mathrm{p}=0.019^{*}$ & $0.29(0.002-3.86)$ & ${ }^{\mathrm{Mw}} \mathrm{p}=0.95$ \\
\hline & $\mathrm{L} 2 / 3$ & $32.68(2.36-250.32)$ & & $8.28(0.59-42.67)$ & & $0.45(0.02-2.03)$ & \\
\hline \multirow{2}{*}{$\begin{array}{l}\text { Pathological } \\
\text { grade }\end{array}$} & Low & $20.93(4.11-75.46)$ & $\mathrm{Mw}_{\mathrm{p}}=0.251$ & $6.89(3.26-62.05)$ & $\mathrm{MW}_{\mathrm{p}}=0.402$ & $0.17(0.03-0.85)$ & ${ }^{\mathrm{MW}} \mathrm{p}=0.95$ \\
\hline & High & $40.59(2.36-426.87)$ & & $5.22(0.59-42.67)$ & & $0.45(0.002-3.86)$ & \\
\hline \multirow{2}{*}{$\begin{array}{l}\text { Mucinous } \\
\text { component }\end{array}$} & Present & $65.24(6.86-250.32)$ & ${ }^{\mathrm{Mw}} \mathrm{p}=0.288$ & $1.44(0.59-13.5)$ & ${ }^{\mathrm{MW}} \mathrm{p}=0.022 *$ & $1.68(1.10-3.86)$ & ${ }^{\mathrm{MW}} \mathrm{p}<0.001^{*}$ \\
\hline & Absent & $30.65(2.36-426.87)$ & & $5.6(0.89-62.05)$ & & $0.21(0.002-1.08)$ & \\
\hline \multirow[t]{2}{*}{ MRF } & MRF- & $16.42(2.36-53.73)$ & ${ }^{\mathrm{MW}} \mathrm{p}<0.001 *$ & $5.6(0.89-62.05)$ & ${ }^{\mathrm{MW}} \mathrm{p}=0.28$ & $0.21(0.02-1.16)$ & ${ }^{\mathrm{Mw}} \mathrm{p}=0.103$ \\
\hline & MRF+ & $86.68(24.04-426.87)$ & & $5.05(0.59-36.64)$ & & $0.85(0.002-3.86)$ & \\
\hline \multirow[t]{3}{*}{$\mathrm{cT}$} & $\mathrm{cT} 1 / 2$ & $9.39(2.36-53.73)^{a^{* * *}}$ & ${ }^{\mathrm{KW}} \mathrm{p}=0.002 *$ & $7.25(0.89-42.67)$ & ${ }^{\mathrm{KW}} \mathrm{p}=0.749$ & $0.18(0.02-1.16)$ & ${ }^{\mathrm{KW}} \mathrm{p}=0.158$ \\
\hline & cT3 & $29.23(4.11-213.43)^{a^{* * *}}$ & & $4.96(0.59-62.05)$ & & $0.41(0.002-1.68)$ & \\
\hline & cT4 & $177.71(24.04-426.87)^{\mathrm{b} * *}$ & & $6.62(0.6-36.64)$ & & $0.88(0.09-3.86)$ & \\
\hline \multirow[t]{2}{*}{$\mathrm{cN}$} & $\mathrm{cN} 0$ & $11.94(2.41-53.73)$ & ${ }^{\mathrm{MW}} \mathrm{p}<0.001 *$ & $8.37(1.55-62.05)$ & ${ }^{\mathrm{MW}} \mathrm{p}=0.103$ & $0.52(0.02-1.16)$ & ${ }^{\mathrm{MW}} \mathrm{p}=0.536$ \\
\hline & $\mathrm{cN} 1 / 2$ & $65.69(2.36-426.87)$ & & $5.05(0.59-36.64)$ & & $0.31(0.002-3.86)$ & \\
\hline \multirow[t]{2}{*}{ cTNM } & Stage II & $11.94(2.36-53.73)$ & ${ }^{\mathrm{MW}} \mathrm{p}<0.001 *$ & $6.75(0.89-62.05)$ & ${ }^{\mathrm{MW}} \mathrm{p}=0.233$ & $0.21(0.02-1.16)$ & ${ }^{\mathrm{Mw}} \mathrm{p}=0.217$ \\
\hline & Stage III & 75.46 (24.04- 426.87) & & $5.05(0.59-36.64)$ & & $0.38(0.002-3.86)$ & \\
\hline
\end{tabular}

MWp, $\mathrm{p}$ value for Mann Whitney test; KWp, $\mathrm{p}$ value for Kruskal Wallis test; $*$, Statistically significant at $\mathrm{p} \leq 0.05 ; * *$, Medians with differing superscripts within columns are significantly different at the adjusted $\mathrm{p} \leq 0.05$ based on post hoc paired comparison.

noted as regards sex of the patients $(\mathrm{p}=0.51)$ nor the location of the rectal tumors $(\mathrm{p}=0.95)$. Furthermore, rectal tumors with different pathological grades of differentiation did not show statistically significant difference in $A T O H 1$ expression levels $(\mathrm{p}=0.95)$. Conversely, presence of mucinous component in rectal adenocarcinomas was a highly significant factor correlating with $A T O H 1$ fold change, where median ATOH1 expression level was significantly higher in rectal adenocarcinomas with mucinous components compared to non-mucinous tumors $(\mathrm{p}<0.001)$. Finally, no statistically significant difference was observed between $A T O H 1$ fold change and MRF involvement $(\mathrm{p}=0.103)$, depth of tumor invasion ( $p=0.158)$, spread to regional LN $(p=0.536)$ nor clinical staging $(p=0.217)$ of rectal tumors (Table 2$)$.

Correlation analysis revealed highly significant
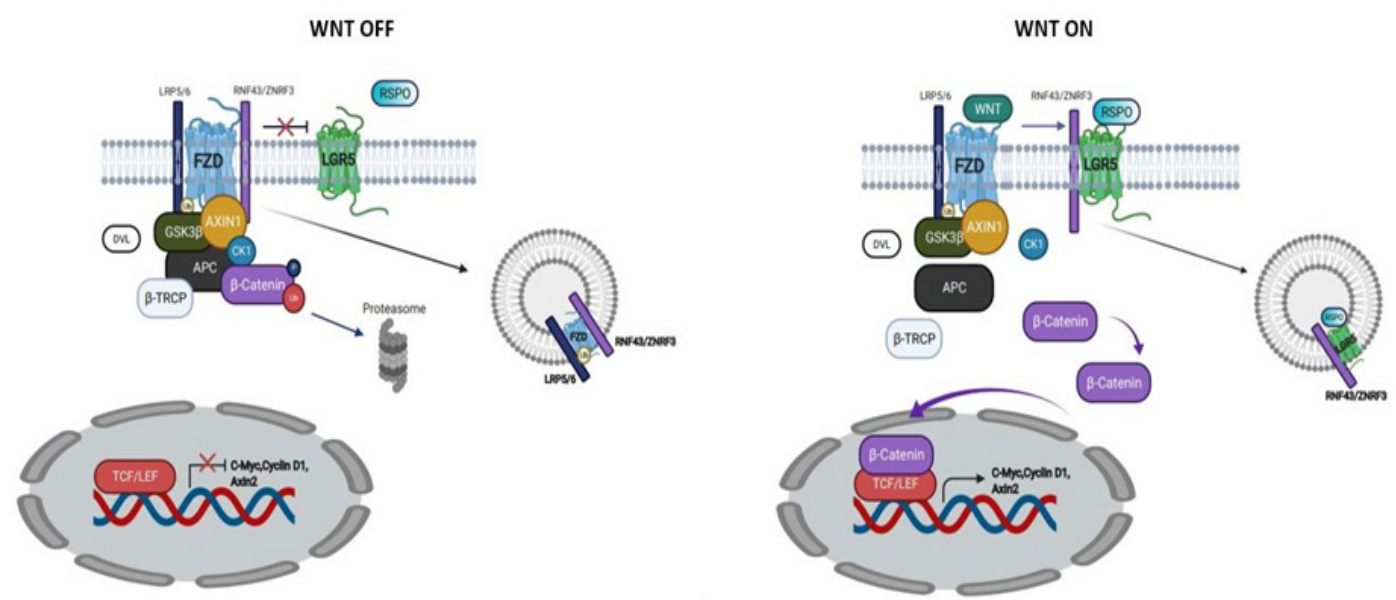

Figure 1. WNT OFF, Without LGR5/RSPO Complex, Two Transmembrane E3 Ligases (RNF43 and ZNRF3) Remove the Wnt Receptors from the Cell Membrane, Internalize and Degrade them. WNT ON, The binding of RESPO to LGR5 neutralize the transmembrane ligases, they cannot remove the Wnt receptors from the cell surface. FZD and LRP5/6 binds Wnt ligands leading to stabilized $\beta$-catenin. 
Table 3. Relative Gene Expression in RC Patients in Relation to Response to Neoadjuvant CRT

\begin{tabular}{lccc}
\hline Relative gene expression & Responders & Non-responders & Statist. Sig. \\
\hline $\begin{array}{l}\text { LGR5 } \\
\text { Median (Min-Max) }\end{array}$ & $11.58(2.36-53.73)$ & $70.58(24.04-426.87)$ & $\mathrm{p}<0.001^{*}$ \\
$\begin{array}{l}\text { HES1 } \\
\text { Median (Min-Max) }\end{array}$ & $5.28(0.59-36.64)$ & $5.50(0.89-62.05)$ & $\mathrm{p}=0.498$ \\
$\begin{array}{l}\text { ATOH1 } \\
\quad \text { Median (Min-Max) }\end{array}$ & $0.45(0.002-3.86)$ & $0.19(0.02-1.16)$ & $\mathrm{p}=0.142$ \\
\hline
\end{tabular}

$\mathrm{p}, \mathrm{p}$ value for Mann Whitney test. *, Statistically significant at $\mathrm{p} \leq 0.05$.

association between $L G R 5$ expression levels and response to CRT among the studied RC patients ( $\mathrm{p}<0.001)$. LGR5 was substantially over expressed among non- responders (SD \& PD) to CRT as it ranged from 24.04- 426.87 with a median of 70.58 . On the contrary, responders (CR \&PR) to CRT showed much lower levels of LGR5 expression, ranging from 2.36 to 53.73 with a median of 11.58. Nevertheless, response to neoadjuvant CRT was not associated with $H E S 1$ nor $A T O H 1$ relative expression levels ( $\mathrm{p}=0.498, \mathrm{p}=0.142$, respectively) (Table 3, Figure 3 ).

Moreover, ROC curve analysis for expression of LGR5 and response to neoadjuvant CRT showed that $L G R 5$ expression can significantly discriminate between responders and non- responders by $95 \%$ accuracy (AUC $=0.95, \mathrm{p}<0.001)$. This indicated that the LGR5 expression level is an excellent predictive factor for response among RC patients with high sensitivity (92.86\%) and specificity (87.5\%). A cut-off value of 30.65 best distinguished between responder and non- responder RC patients (Figure 5).

\section{Discussion}

The current work studied gene expression of 3 CSCs related genes, namely LGR5, HES1, and ATOH1 in rectal adenocarcinomas among young Egyptian patients.

LGR 5 median expression level in rectal tumor tissues was significantly higher than paired normal tissues (Table 1). Increased protein and mRNA expression of LGR5, using qPCR or Immunohistochemistry (IHC), have been previously reported in CRC tissues compared to normal mucosa. (Uchida et al., 2010; He et al., 2014; Kleist et al., 2011; Hsu et al., 2013) Overexpression of LGR5 in CRC maybe attributed to enrichment of 'stem-like' cancer cells, upregulation of the Wnt signaling pathway and/or Wnt -dependent maintenance of stemness (Uchida et al., 2010, Walker et al., 2011). It was suggested that transformed LGR5-positive stem cells are the origin of cancer in the intestine (Hsu et al., 2013; Yui et al.; 2012). Therefore, LGR5 was proposed as an ideal marker for CSCs in CRC (Kemper et al., 2012; Hirsch et al., 2014).

On the other hand, few studies put forward

\section{Signal sending-cell}
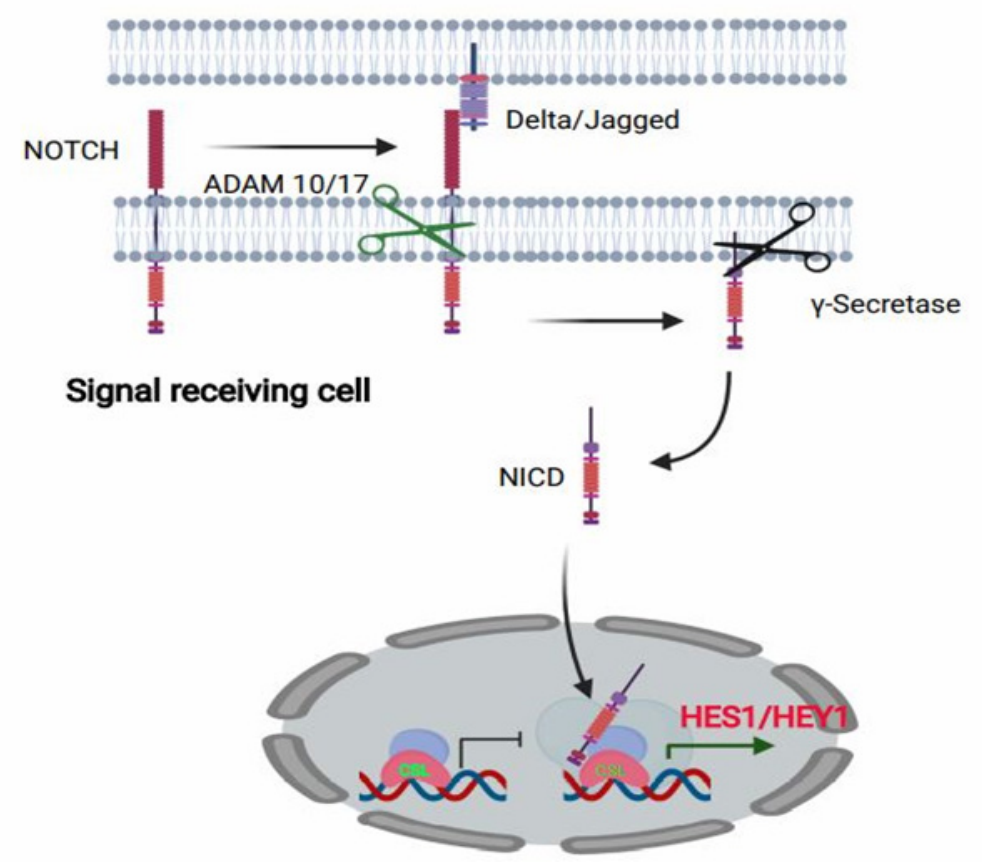

Figure 2. Notch Signaling and the Maintenance of CSC. Two Nearby cells, one signal sending and other signal receiving cell interact. The binding of Delta/Jagged to the Notch leads to S2 cleavage by ADAM10 or 17, which is followed by S3 cleavage by $\gamma$-secretase. The S3 cleavage gives rise to an intracellular Notch fragment (NICD) that migrates into the nucleus where it binds to a complex of protein, leading to the de-repression of transcription of hair/ enhancer of split (Hes) and Hey. 


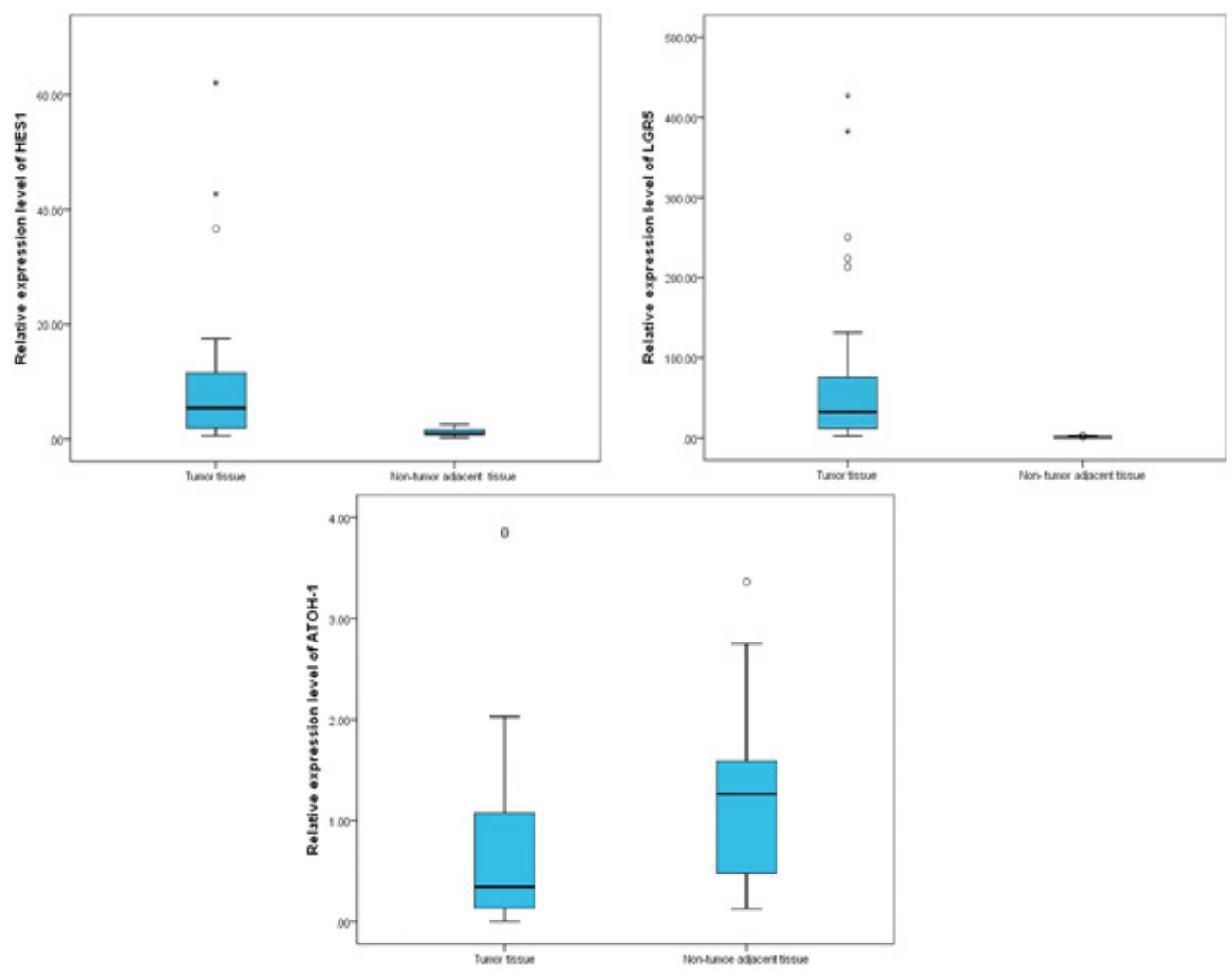

Figure 3. Box Plot Showing Expression of LGR5, HES1\& ATOH1 in Rectal Tumor and Non-tumor Adjacent tissues. The upper and lower borders of the box represent $25^{\text {th }}$ and $75^{\text {th }}$ percentile, the horizontal line inside the box represents the median $\left(50^{\text {th }}\right)$. Circles represent the outliers. Data were analyzed using Mann-Whitney test.
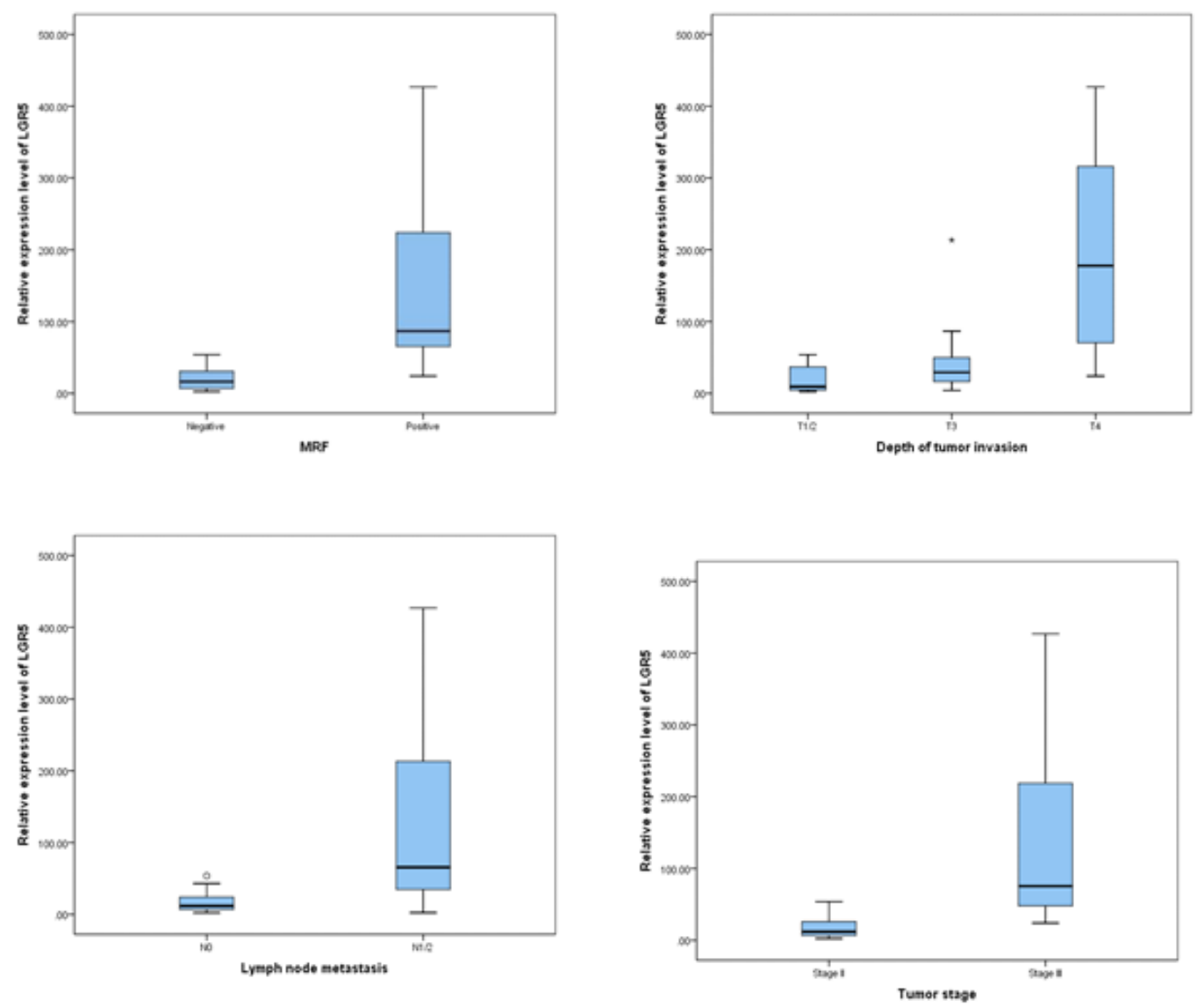

Figure 4. Correlation between LGR5 Expression Levels and MRF Involvement, Depth of Tumor Invasion, LN Metastasis and Clinical Tumor Staging. 

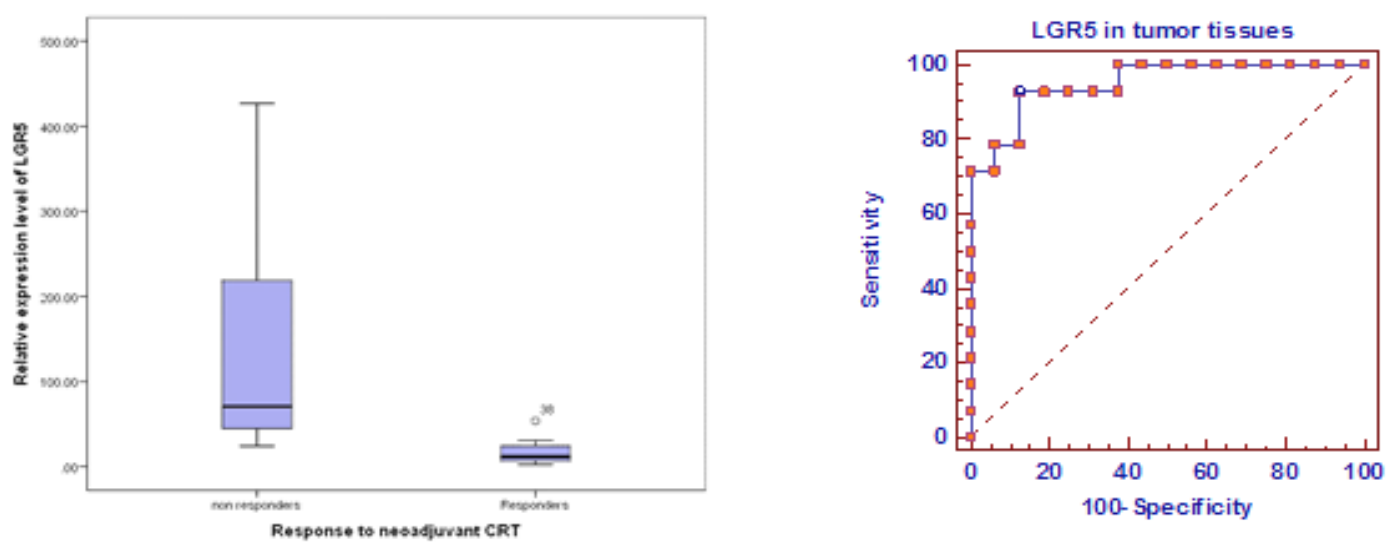

Figure 5. Response to Neoadjuvant CRT Based on RECIST 1.1 Guidelines in Relation to LGR5 Relative Expression among the studied RC patients. A, Box plot showing LGR5 over expression among non- responders to CRT compared to responders. B, ROC curve analysis indicating that LGR5 expression is an excellent predictive factor for response to neoadjuvant CRT.

the opposite points, where increased invasion and tumorigenicity, besides enhanced Wnt signaling were observed upon knockdown of $L G R 5$ in a xenograft model (Walker et al., 2011). This remark is in agreement with the other data suggesting $L G R 5$ as a negative regulator of canonical Wnt pathways (Garcia et al., 2009; Wu et al., 2014). Such discrepancy suggests that LGR5 and its ligands R-spondin might have opposing roles in different contexts which remain to be elucidated. Another possible explanation is that these studies observed a negative feedback loop where LGR5 expression keeps in check over-activation of canonical Wnt signaling (Walker et al., 2011). Moreover, the discrepancies between different studies, could be attributed also to the fact that LGR5 expression can be, alternatively or additionally, stimulated by other mechanisms than Wnt signaling (Sancho et al., 2009; Tanese et al., 2008). Another hypothesis of relation between $L G R 5$ expression and Wnt pathway was proposed by two reports, which supported a bi-modal regulation of $L G R 5$ expression by Wnt signaling: induction of $L G R 5$ at medium levels of Wnt activation but loss of expression with higher levels of Wnt activation (Sun et al., 2009; Lewis et al., 2010).

The present study next investigated HES1 gene expression as a marker for Notch signaling. Results displayed that HES1 mRNA levels were significantly elevated in rectal tumor relative to normal tissues (Table 1). Comparable results of HES1 mRNA overexpression were observed by several studies (Jin et al., 2012; Yuan et al., 2015; Candy et al., 2013; Meng et al., 2007). In primary CRC, Notch signaling was shown to be strongly activated, and has an important role in cancer initiation and progression (Qiao et al., 2009).

The current work studied, as well, the expression of ATOH (Kong et al., 2001). ATOH1 mRNA expression was significantly downregulated in rectal tumor tissues compared to normal tissues, with very low levels of ATOH1 expression noted among most of malignant tissues (Table 1). A growing number of studies further confirmed our results and supported a tumor suppressive function of ATOH1( Leow et al., 2005; Park et al., 2006; Bossuyt et al., 2009). It was suggested that tumor suppressive function of ATOH1 is mediated by its effects on CSCs (Kazanjian et al., 2010). In CRC, several mechanisms work to skew the normal Notch- $A T O H 1$ balance to favor Notch activation, including silencing of $A T O H 1$ by genetic and epigenetic mechanisms (Bossuyt et al., 2009).

In the current study, correlation analysis demonstrated that LGR5 expression in human RC clinical specimens was significantly correlated with depth of tumor invasion, LN metastasis, cTNM stage and MRF involvement, but it was not correlated with gender, age, tumor site, nor pathological features (Table 2). Our results suggest that high expression levels of LGR5 are correlated with more malignant tumors. This is in agreement with a meta-analysis by Jiang et al., 2015 who reported that LGR5 overexpression is significantly associated with deep invasion, LN metastasis, distant metastasis and AJCC stage, however, it was not correlated with tumor grade (Jiang et al., 2016). Their results proposed LGR5 expression as a prognostic factor for CRC patients. Correspondingly, Han et al.,2015 meta-analysis, showed almost equivalent results (Han et al., 2015). Nevertheless, Yang et al., 2016 meta-analysis, detected correlation between LGR5 expression and TNM stage only (Yang et al., 2016). Studying the association of LGR5 with clinical outcome in RC after preoperative CRT, Saigusa et al.,2012, reported also that elevated $L G R 5$ expression was significantly correlated with poor recurrence-free and Overall Survival (Saigusa et al., 2012). Other groups had concluded that $L G R 5$ expression was involved in the CRC progression, as well (Uchida et al., 2010; Hsu et al., 2013, Ong et al., 2016).

The most well established correlations with LGR5 expression encountered in the aforementioned studies (Uchida et al., 2010; Han et al., 2015; Wu et al., 2016) are in accordance with our results. Overall, these findings indicate that LGR5 gene expression association with 
clinical findings among young onset RC patients, are in line with gene expression patterns encountered in previous studies conducted in older age groups.

On the other hand, Ziskin et al., (2013) showed that $L G R 5$ expression, was not associated with increased tumor aggressiveness and was not a prognostic factor for CRC. Surprisingly, it was also reported that methylation of CSCs associated Wnt target genes predicts poor prognosis in CRC patients, hence high LGR5 expression is associated with a favorable prognosis (De Sousa et al., 2011).

According to our results, MRF involvement was positively correlated with LGR5 expression level. (Table 2). Involvement of or close proximity to the MRF preoperatively increases the risk of compromised Circumferential Resection Margin (CRM) after surgery, which was repeatedly defined as an independent predictor of a poor outcome.(Quirke et al., 2009, Park et al., 2014). Hence, such correlation with high $L G R 5$ expression is not unexpected and in line with the main assumption of unfavorable impact of high $L G R 5$ expression.

On the other hand, no correlations were found with gender and pathological features according to the current work. Previous study suggested that the role of LGR5 might be different between male and female patients in tumorigenesis (Hsu et al., 1998). However, previous meta-analysis, Han et al., (2015) showed that LGR5 was not obviously correlated with the gender of patients. Regarding relation to pathological criteria, previous results are inconsistent (Wu et al., 2012; Jiang et al., 2016; Jiang et al., 2015; Yang et al., 2016).

Correlations between LGR5 expression in primary tumors from CRC patients and different clinicopathological features are inconsistent. Many factors may have influenced such discrepancy in results. For example, additional subgroup analysis in previous meta-analysis, (Chen et al., 2014) revealed that many factors influenced the significance of the correlation between LGR5 expression and prognosis in CRC patients. Hence, the discrepancy in the correlation results could be attributed to relatively small number of patients in some of the studies including the present study, and the dissimilarity of patient populations in different studies. It was observed that high $L G R 5$ expression was significantly associated with poor outcome in the studies conducted on Asian patients, but not in non- Asians (Chen et al., 2014; Jiang et al., 2015). Therefore, further investigations were recommended to verify whether the prognostic value of $L G R 5$ in CRC is associated with the variation of the study population. This observation further elucidates the importance of our research on Egyptian patients. The diversity of techniques used to detect expression of LGR5 maybe another factor contributing to heterogeneity of results in different studies. Most of the studies primarily depend on IHC, which is highly altered by methodological factors. Another potential mechanism to the opposing results is variants of the LGR5 gene (Kleist et al., 2012; Szkandera et al., 2015).

Aiming to explore the clinical significance of Notch signaling in RC, the possible relation between HES1 and ATOH1 expression levels and various clinicopathological characteristics of rectal tumors were studied to evaluate their prognostic potential. HES1 and ATOH1 expression levels did not show significant association with most of the clinicopathological criteria of the studied RC patients. Yet, both HES1 and ATOH1 expression levels were significantly correlated with presence of mucinous component in rectal adenocarcinomas (Table 2).

Many studies have recently focused on the role of Notch signaling pathway in influencing the differentiation decision of cells in the GIT. A previous study agreed with our results, where overexpression of Notch transcription factors, including HES1 was not associated with tumor differentiation (Candy et al., 2013). Nevertheless, other studies indicated that the expression levels of HES1 were associated with the pathological tumor type and degree of differentiation (Jin et al., 2012; Gao et al., 2014; Ahadi et al., 2016). As regards to ATOH1, a study on mesenchymal GIT tumor was concordant with our results (Huang et al., 2014).

Clinical study of HES1 overexpression in CRC have shown that the HES1 expression was not associated with survival (Reedijk et al., 2008). In accordance with our results, activation of Notch signaling was noted in human CRC, but the level of signaling is not prognostic. These findings were further confirmed later (Candy et al., 2013). Nevertheless, other studies showed contradictory results, where high HES1 expression was associated with better or poor prognosis (Yuan et al., 2015; Ahadi et al., 2016). Although HES1 is considered the most well characterized downstream target of Notch pathway, HES1 can also be elevated via other signals (Ingram et al., 2008; Stockhausen et al., 2005). This may partly explain the inconsistent results on the prognostic significance of HES1 in previous studies. In addition, the diverse heterogeneity of tumor samples included in different studies may further add to discrepancy in results. Therefore, the association and prognostic value of HES1 in CRC remains to be investigated in future studies.

Regarding the association of clinical findings with ATOH1 expression, the present work didn't find significant correlation between ATOH1 mRNA levels and various tumor criteria (Table 2). A number of studies have suggested that loss of $A T O H 1$ strongly enhances the formation and progression of different types of tumors and was identified as independent predictive factors for poor outcome (Han et al., 2015; Huang et al., 2014). In CRC, because the expressed $A T O H 1$ protein is degraded, its function has not been elucidated in details since most of studies relies on IHC analysis.

Mucinous cancers (MC) are distinct classes of CRC, with unique genetic and epigenetic alterations (Song et al., 2005; Bosman et al., 2010). MC may exhibit more aggressive behavior and a worse prognosis than their nonmucinous counterparts. (Yamamoto et al., 1993). In the present study, HES1 and ATOH1 were inversely expressed in rectal adenocarcinomas with mucinous component (Table 2). Previous studies have demonstrated that cell fate in human gut epithelium seems to be directly controlled by two main Notch target genes: HES1 and ATOH1 (Fre et al., 2005). Hence, the pattern of clinical expression noted in our results is consistent with molecular understanding of Notch signaling. Results of animal studies revealed that 
intestines of Math1 (mouse homolog of human ATOH1) null mice fail to develop secretory cell lineages (Yang et al., 2001). The role of ATOH1 in mucinous cancer is far beyond just induction of differentiation. Microarray analysis showed attainment of more malignant potential by $A T O H 1$ protein stabilization, suggesting the mechanism by which $\mathrm{MC}$ are more malignant than non-mucinous adenocarcinoma (Kano et al., 2013).

In the present study, gene expression data showed that patients with lower levels of LGR5 expression had significantly better response than patients with higher levels of $L G R 5$ expression (Table 3 ). An evidence on the predictive power of $L G R 5$ expression was demonstrated by previous research showing better response to 5-FU based treatment among patients with low LGR5 level compared to those with high level. This finding agrees with our study, although in different patient cohort, since our patients received Capecitabine (5FU based treatment) (Hsu et al., 2013). Furthermore, it was reported that RC specimens from patients with poor pathological response had significantly higher LGR5 expression levels than those exhibiting a positive response after CRT. Thus, it was suggested that $L G R 5$ expression may be implicated in resistance to CRT in RC (Saigusa et al., 2012).

On the contrary, Planutis et al 2015, (Planutis et al., 2015) in their work hypothesized that LGR5-expressing cells would be more chemotherapy sensitive, as LGR5 is usually a marker of dividing cells. They concluded that CRC cells that express LGR5 are more sensitive to the chemotherapeutic compounds Irinotecan and Oxaliplatin, but not to 5-FU. They proposed that LGR5 makes cells vulnerable to chemotherapy by increasing their propensity to divide through activation of the Wnt/ $\beta$-catenin pathway. Since genetic polymorphism in LGR5 gene was suggested as predictive biomarker for response to FU in CRC patients, variants of LGR5 gene is one factor which might explain this discrepancy between different studies (Szkandera et al., 2015).

The molecular mechanisms underlying LGR5-associated chemoresistance were investigated by Liu et al 2013 (Hsu et al., 2013). They illustrated that elevated LGR5 caused resistance to 5-FU and Oxaliplatin, which was associated with high ABCB1 expression, an efflux pump for chemotherapeutic drugs. The limited success of chemotherapy may be also due to the failure of current therapies to effectively kill CSCs. Remarkably, it was reported that after anticancer agent treatment, some fast-proliferating LGR5-positive CSCs converted to slow proliferating $L G R 5$-negative cells and entered quiescence to escape chemotherapy-mediated killing. After the drug removal, these LGR5-negative cells reverted to the $L G R 5$-positive state to reconstitute the entire tumor, suggesting a pool of CSCs with the ability to interconvert between two distinct states (Kobayashi et al., 2012). The authors proposed that the ability of CSCs to switch between these two states might explain how some rare CSCs survive during drug therapy (Kobayashi et al., 2012).

Altogether, these notions support our observation that high LGR5 expression level was associated with resistance to neoadjuvant CRT in young RC patients, and that early onset $\mathrm{RC}$ did not show difference in the role of $L G R 5$ as a predictive marker of chemoresistance. Therefore, $L G R 5$ is an attractive target for combating chemoresistance in RC.

Given its role in cellular proliferation and CRC tumorigenesis and progression, the present study investigated whether tumor expression of Notch target genes, HES1 and ATOH1, may be used as predictors of response to CRT in RC patients. However, no statistically significant correlation was detected between HES1 nor ATOH1 and response to CRT. (Table 3). In CRC, few studies have addressed the role of HES1 in response to chemotherapy. It was shown that 5-FU, Oxaliplatin and Irinotecan-induced chemoresistance in CRC cells was promoted by Notch transcription factors, such as HES1, and abrogated using Notch inhibitory therapy using Gamma -Secretase Inhibitors (GSI) (Meng et al., 2007). A clinical study, reported that the combination of HEY1, HES1 and SOX9 protein overexpression were predictive of poorer response to chemotherapy in CRC patients (Candy et al., 2013). As the included cohort of patients in our study didn't show different responses to CRT in relation to different HES1 expression levels, this may be due to lack of enough samples or the variation in age and site of tumors in our patients. Hence, additional studies seem to be needed especially comparing young and old RC patients in regard to the correlation between HES1 expression and chemoresistance.

The role of $A T O H 1$ in chemoresistance in CRC patients was the point of investigation in a few recent studies. It was revealed that cells transducing ATOH1 showed chemoresistance against Oxaliplatin. Interestingly, it was reported that Oxaliplatin stabilized $A T O H 1$ protein, resulting in the suppression of the apoptosis signaling. Induction of CSCs and mucinous phenotypes, by ATOH1 protein stabilization, leading to chemoresistance, was described, as well. Another interesting result was that the ATOH1 protein extended the G0/G1 phase of the cell cycle and led to avoidance of G2 phase entry, which most of alkylating agents target (Kano et al., 2013). These results are in concordant with findings observed in a later study (Fukushima et al., 2015). Collectively, these findings illustrated the acquisition of chemoresistance by $\mathrm{MC}$ that express the ATOH1 protein. However, almost all these studies were conducted on MC. Unfortunately, our study didn `t follow this model as our cohort included both mucinous and non-mucinous rectal adenocarcinomas, with only few tumors showing mucinous components. Hence this might have hindered attainment of significant results on ATOH1 expression relation to response to CRT.

In conclusion, the current study suggested that $L G R 5$ may not only serve as a novel prognostic indicator in young RC patients, but may also be an excellent predictor for response to CRT. Additionally, the molecular characterization of rectal adenocarcinomas with mucinous components revealed high ATOH1 expression levels, adding supplementary evidence to its critical effect on cellular differentiation. However, HES1 and ATOH1 expressions were not prognostic nor predictive of outcome or response to CRT. Overall, our findings indicate that LGR5, HES1 and ATOH1 gene expression patterns among young onset RC patients, are in line with patterns 
encountered in older age groups. It is noteworthy that the current study is, to the best of our knowledge, the first Egyptian study to address the molecular features of young RC patients by studying CSCs related genes.

However, data in this study should be interpreted with some caution, because the baseline characteristics of patients might have affected the conclusions of each of the studied genes, including the sample size, follow-up period and clinical stage, among other aspects. Hence, a larger study population, inclusion of both young and old Egyptian RC patients, with a long-term follow-up is needed to validate these results. Besides, putting in consideration the known predictive and prognostic factors, such as MMR deficiency, is highly suggested. One more limitation may be due to the functional redundancy of many signaling molecules and the strong interaction between signaling pathways which have diverse effects on downstream gene expression.

Finally, the CSCs hypothesis may herald a paradigm shift in oncologic diagnosis and treatment. We are only beginning to understand the multifaceted roles of CSCs in RC. Further studies will reveal more detailed mechanisms by which CSCs contribute to intestinal tumor progression, hence better characterize RC molecular prognostic markers. Research directed towards identification and treatment of CSCs, may eventually lead to better screening, early detection, treatment, and prognosis of $\mathrm{RC}$ patients.

\section{Author Contribution Statement}

1. Waleed Arafat- conceived, designed the experiments and acquired the funding. 2. Heba Morsy, Ahmed Gaballah, Mohamed Samir- did the data collection. 3. Heba Morsy, Mohamed Shamseya, Hanan Mahrous, Abeer Ghazal, Mervat Hashish. -contributed to specific areas of the methods, data analysis, statistics, and quality control. 4. Waleed Arafat, Heba Morsy- analyzed the data 5. Vandrome Nakundi, Heba Morsy: wrote the first draft of the manuscript and conceived the diagrams. All authors read and approved the final manuscript.

\section{Acknowledgments}

Funding Statement

- STDF Grant for Waleed Arafat (17-144-Alex Rep2015)

- Support of the NGO Förderverein Uni Kinshasa e.V and Else-Kroener-Fresenius Stiftung

\section{Conflicts of interest}

The authors report no conflicts of interest

\section{References}

Ahadi M, Andrici J, Sioson L et al (2016). Loss of Hes1 expression is associated with poor prognosis in colorectal adenocarcinoma. Hum Pathol, 57, 91-7.

Ahmed MAH, Al-Attar A, Kim J, et al (2009). CD24 shows early upregulation and nuclear expression but is not a prognostic marker in colorectal cancer. J Clin Pathol, 62, 1117-22.
Baker AM, Graham TA, Elia G, Wright NA, Rodriguez-Justo M (2015). Characterization of LGR5 stem cells in colorectal adenomas and carcinomas. Sci Rep, 5, 1-8.

Barker N (2014). Adult intestinal stem cells: Critical drivers of epithelial homeostasis and regeneration. Nat Rev Mol Cell Biol, 15, 19-33.

Bosman FT, Carneiro F, Hruban RH, et al (2010). WHO classification of tumours of the digestive system. 4th ed. World Health Organization. Geneva, Switzerland, pp 417.

Bossuyt W, Kazanjian A, De Geest N, et al (2009). Atonal homolog 1 is a tumor suppressor gene. PLoS Biol, 7, 0311-26.

Candy PA, Phillips MR, Redfern AD et al (2013). Notchinduced transcription factors are predictive of survival and 5-fluorouracil response in colorectal cancer patients. $\mathrm{Br} \mathrm{J}$ Cancer, 109, 1023-30.

Chen J, Imanaka N, Chen J, Griffin JD (2010). Hypoxia potentiates Notch signaling in breast cancer leading to decreased E-cadherin expression and increased cell migration and invasion. Br J Cancer, 102, 351-60.

Chen K, Huang YH, Chen JL (2013). Understanding and targeting cancer stem cells: Therapeutic implications and challenges. Acta Pharmacol Sin, 34, 732-40.

Chen Q, Zhang X, Li WM, et al (2014). Prognostic value of LGR5 in colorectal cancer: A meta-analysis. PLoS One, 9.

Choi D, Lee HW, Hur KY et al (2009). Cancer stem cell markers CD133 and CD24 correlate with invasiveness and differentiation in colorectal adenocarinoma. World $J$ Gastroenterol, 15, 2258-64.

De Sousa E Melo F, Colak S, Buikhuisen J, et al (2011). Methylation of cancer-stem-cell-associated wnt target genes predicts poor prognosis in colorectal cancer patients. Cell Stem Cell, 9, 476-85.

Eisenhauer EA, Therasse P, Bogaerts J, et al (2009). New response evaluation criteria in solid tumours: Revised RECIST guideline (version 1.1). Eur J Cancer, 45, 228-47.

Fre S, Huyghe M, Mourikis P, et al (2005). Notch signals control the fate of immature progenitor cells in the intestine. Nature, 435, 964-8.

Fukushima K, Tsuchiya K, Kano Y, et al (2015). Atonal homolog 1 protein stabilized by tumor necrosis factor $\alpha$ induces high malignant potential in colon cancer cell line. Cancer Sci, 106, 1000-7.

Gao F, Zhang Y, Wang S et al (2014). Hes1 is involved in the self-renewal and tumourigenicity of stem-like cancer cells in colon cancer. Sci Rep, 4.

Garcia MI, Ghiani M, Lefort A, et al (2009). LGR5 deficiency deregulates Wnt signaling and leads to precocious Paneth cell differentiation in the fetal intestine. Dev Biol, 331, 58-67.

Han Y, Xue X, Jiang M, et al (2015) . LGR5, a relevant marker of cancer stem cells, indicates a poor prognosis in colorectal cancer patients: A meta-analysis. Clin Res Hepatol Gastroenterol, 39, 267-73.

He S, Zhou H, Zhu X, et al (2014). Expression of Lgr5, a marker of intestinal stem cells, in colorectal cancer and its clinicopathological significance. Biomed Pharmacother, 68, 507-13.

Hirsch D, Barker N, Mcneil N, et al (2014). LGR5 positivity defines stem-like cells in colorectal cancer. Carcinogenesis, 35, 849-58.

Horst D, Scheel SK, Liebmann S, et al (2009). The cancer stem cell marker CD133 has high prognostic impact but unknown functional relevance for the metastasis of human colon cancer. $J$ Pathol, 219, 427-34.

Hsu HC, Liu YS, Tseng KC, et al (2013). Overexpression of Lgr5 correlates with resistance to 5-FU-based chemotherapy in 
colorectal cancer. Int J Colorectal Dis, 28, 1535-46.

Hsu SY, Liang SG, Hsueh AJW (1998). Characterization of two LGR genes homologous to gonadotropin and thyrotropin receptors with extracellular leucine-rich repeats and a $\mathrm{G}$ protein-coupled, seven-transmembrane region. $\mathrm{Mol}$ Endocrinol, 12, 1830-45.

Huang H, Zhai X, Zhu H, et al (2014). Upregulation of Atoh1 correlates with favorable survival in gastrointestinal stromal tumor. Int J Clin Exp Pathol, 7, 7123-30.

Ibrahim AS, Khaled HM, Mikhail NN, Baraka H, Kamel H (2014). Cancer incidence in Egypt: Results of the national population-based cancer registry program. J Cancer Epidemiol, 2014

Ingram WJ, McCue KI, Tran TH, Hallahan AR, Wainwright BJ (2008). Sonic Hedgehog regulates Hes1 through a novel mechanism that is independent of canonical Notch pathway signalling. Oncogene, 27, 1489-500.

Jang BG, Kim HS, Chang WY, et al (2018). Expression profile of LGR5 and its prognostic significance in colorectal cancer progression. Am J Pathol, 188, 2236-50.

Jiang Y, Li W, He X, et al (2015). Lgr5 expression is a valuable prognostic factor for colorectal cancer: Evidence from a meta-analysis. BMC Cancer, 15, 1-10.

Jiang Y, Li W, He X, et al (2016). Lgr5 expression is a valuable prognostic factor for colorectal cancer: Evidence from a meta-analysis. BMC Cancer, 16, 1-9.

Jin HY, Zhang HY, Wang X, Xu J, Ding Y (2012). Expression and clinical significance of Notch signaling genes in colorectal cancer. Tumor Biol, 33, 817-24.

Kano Y, Tsuchiya K, Zheng X, et al (2013). The acquisition of malignant potential in colon cancer is regulated by the stabilization of Atonal homolog 1 protein. Biochem Biophys Res Commun, 432, 175-81.

Kazanjian A, Noah T, Brown D, Burkart J, Shroyer NF (2010). Atonal homolog 1 is required for growth and differentiation effects of Notch $/ \gamma$-secretase inhibitors on normal and cancerous intestinal epithelial cells. Gastroenterology, 139, 918-28.

Kemper K, Prasetyanti PR, De Lau W, et al (2012). Monoclonal antibodies against Lgr5 identify human colorectal cancer stem cells. Stem Cells, 30, 2378-86.

Kleist B, Xu L, Kersten C, et al (2012). Single nucleotide polymorphisms of the adult intestinal stem cell marker Lgr5 in primary and metastatic colorectal cancer. Am J Transl Res, 4, 279-90.

Kleist B, Xu L, Li G, Kersten C (2011). Lgr5 expression in the metastatic cascade of colorectal cancer. Int J Clin Exp Pathol, 4, 327-35.

Kobayashi S, Yamada-Okabe H, Suzuki M, et al (2012). LGR5-positive colon cancer stem cells interconvert with drug-resistant LGR5-negative cells and are capable of tumor reconstitution. Stem Cells, 30, 2631-44.

Kong J, Crissey MAS, Sepulveda AR, Lynch JP (2012). Math1/atoh1 contributes to intestinalization of esophageal keratinocytes by inducing the expression of Muc2 and Keratin-20. Dig Dis Sci, 57, 845-57.

Leeflang MMG, Rutjes AWS, Reitsma JB, Hooft L, Bossuyt PMM (2013). Variation of a test's sensitivity and specificity with disease prevalence. CMAJ, 185, 537-44.

Leow CC, Polakis P, Gao WQ (2005). A role for Hath1, a bHLH transcription factor, in colon adenocarcinoma. Ann N Y Acad Sci, 105, 174-83.

Lewis A, Segditsas S, Deheragoda M, et al (2010). Severe polyposis in Apc1322T mice is associated with submaximal Wnt signalling and increased expression of the stem cell marker Lgr5. Gut, 59, 1680-6.

Livak KJ, Schmittgen TD (2001). Analysis of relative gene expression data using real-time quantitative PCR and the $2^{-\Delta \Delta C T}$ method. Methods, 25, 402-8.

Meng R, Shelton C, Cheema H, Li Y, Schwartz G (2007). Gamma-secretase inhibitors abrogate oxaliplatin-induced activation of the Notch-1 signaling pathway in colon cancer cells resulting in enhanced chemosensitivity. Cancer Res, 67, 5742 .

Nimmakayala RK, Batra SK, Ponnusamy MP (2019). Unraveling the journey of cancer stem cells from origin to metastasis. Biochim Biophys Acta Rev Cancer, 1871, 50-63.

Ong CW, Chong PY, McArt DG, et al (2015). The prognostic value of the stem-like group in colorectal cancer using a panel of immunohistochemistry markers. Oncotarget, 6, 12763-73.

Park ET, Oh HK, Gum JR, et al (2006). HATH1 expression in mucinous cancers of the colorectum and related lesions. Clin Cancer Res, 12, 5403-10.

Park JS, Huh JW, Park YA, et al (2014). A circumferential resection margin of $1 \mathrm{~mm}$ is a negative prognostic factor in rectal cancer patients with and without neoadjuvant chemoradiotherapy. Dis Colon Rectum, 57, 933-40.

Planutis AK, Holcombe RF, Planoutene MV, Planoutis KS (2015). SW480 colorectal cancer cells that naturally express Lgr5 are more sensitive to the most common chemotherapeutic agents than Lgr5-negative SW480 cells. Anticancer Drugs, 26, 942-7.

Qiao L, Wong BCY (2009). Role of notch signaling in colorectal cancer. Carcinogenesis, 30, 1979-86.

Quirke P, Steele R, Monson J, et al (2009). Effect of the plane of surgery achieved on local recurrence in patients with operable rectal cancer: a prospective study using data from the MRC CR07 and NCIC-CTG CO16 randomised clinical trial. Lancet, 373, 821-8.

Reedijk M, Pinnaduwage D, Dickson BC, et al (2008). JAG1 expression is associated with a basal phenotype and recurrence in lymph node-negative breast cancer. Breast Cancer Res Treat, 111, 439-48.

Saigusa S, Inoue Y, Tanaka K, et al (2012). Clinical significance of LGR5 and CD44 expression in locally advanced rectal cancer after preoperative chemoradiotherapy. Int J Oncol, 41, 1643-52.

Saigusa S, Tanaka K, Toiyama Y, et al (2010). Immunohistochemical features of CD133 expression: Association with resistance to chemoradiotherapy in rectal cancer. Oncol Rep, 24, 345-50.

Sancho R, Nateri AS, De Vinuesa AG et al (2009). JNK signalling modulates intestinal homeostasis and tumourigenesis in mice. EMBO J, 28, 1843-54.

Siegel RL, Miller KD, Jemal A (2015). Cancer statistics 2015. CA Cancer J Clin, 65, 5-29.

Silla IO, Rueda D, Rodríguez Y, et al (2014). Early-onset colorectal cancer: A separate subset of colorectal cancer. World J Gastroenterol, 20, 17288-96.

Soliman AS, Bondy ML, El-Badawy, et al (2001). Contrasting molecular pathology of colorectal carcinoma in Egyptian and Western patients. Br J Cancer, 85, 1037-46.

Soliman AS, Bondy ML, Levin B, et al (1997). Colorectal cancer in Egyptian patients under 40 years of age. Int J Cancer, 71, 26-30.

Soliman AS, Bondy ML, Raouf AA, et al (1999). Cancer mortality in Menofeia, Egypt: Comparison with US mortality rates. Cancer Causes Control, 10, 349-54.

Song GA, Deng G, Bell I, et al (2005). Mucinous carcinomas of the colorectum have distinct molecular genetic characteristics. Int $J$ Oncol, 26, 745-50.

Srinivasan T, Than EB, Bu P, et al (2016). Notch signalling regulates asymmetric division and inter-conversion between 
lgr5 and bmi1 expressing intestinal stem cells. Sci Rep, 6 , $1-8$.

Stockhausen MT, Sjölund J, Axelson H (2005). Regulation of the Notch target gene Hes-1 by TGF $\alpha$ induced Ras/MAPK signaling in human neuroblastoma cells. Exp Cell Res, 310, 218-28.

Sun X, Jackson L, Dey SK, Daikoku T (2009). In pursuit of leucine-rich repeat-containing G protein-coupled receptor-5 regulation and function in the uterus. Endocrinology, 150, 5065-73.

Szkandera J, Herzog S, Pichler M, et al (2015). LGR5 rs17109924 is a predictive genetic biomarker for time to recurrence in patients with colon cancer treated with 5-fluorouracil-based adjuvant chemotherapy. Pharmacogenomics J, 15, 391-6.

Tanese K, Fukuma M, Yamada T, et al (2008). G-protein-coupled receptor GPR49 is up-regulated in basal cell carcinoma and promotes cell proliferation and tumor formation. $\mathrm{Am} J$ Pathol, 173, 835-43.

Uchida H, Yamazaki K, Fukuma M, et al (2010). Overexpression of leucine-rich repeat-containing $\mathrm{G}$ protein-coupled receptor 5 in colorectal cancer. Cancer Sci, 101, 1731-7.

Vaiopoulos AG, Kostakis ID, Koutsilieris M, Papavassiliou AG (2012). Concise review: Colorectal cancer stem cells. Stem Cells, 30, 363-71.

Visvader JE, Lindeman GJ (2008). Cancer stem cells in solid tumours: Accumulating evidence and unresolved questions. Nat Rev Cancer, 8, 755-68.

Walker F, Zhang HH, Odorizzi A, Burgess AW (2011). LGR5 is a negative regulator of tumourigenicity, antagonizes wnt signalling and regulates cell adhesion in colorectal cancer cell lines. PLoS One, 6.

Wu C, Qiu S, Lu L et al (2014). RSPO2-LGR5 signaling has tumour-suppressive activity in colorectal cancer. Nat Commun, $\mathbf{5}$.

Wu W, Cao J, Ji Z, et al (2016). Co-expression of Lgr5 and CXCR4 characterizes cancer stem-like cells of colorectal cancer. Oncotarget, 7, 81144-55.

Wu XS, Xi HQ, Chen L (2012). Lgr5 is a potential marker of colorectal carcinoma stem cells that correlates with patient survival. World J Surg Oncol, 10, 1.

Yamamoto S, Mochizuki H, Hase K (1993). Assessment of clinicopathologic features of. Am J Surg, 166, 257-61.

Yang L, Lin Y, Liu Z, et al (2016). Impact of LGR5 in colorectal cancer on overall and progression-free survival: A systematic review and meta-analysis. Int J Clin Exp Med, 9, 10537-43.

Yang Q, Bermingham NA, Finegold MJ, Zoghbi HY (2001). Requirement of Math1 for secretory cell lineage commitment in the mouse intestine. Science, 294, 2155-8.

Yasuda H, Tanaka K, Saigusa S, et al (2009). Elevated CD133, but not VEGF or EGFR, as a predictive marker of distant recurrence after preoperative chemoradiotherapy in rectal cancer HIROMI. Oncol Rep, 22, 709-17.

Yuan R, Ke J, Sun L, et al (2015). HES1 promotes metastasis and predicts poor survival in patients with colorectal cancer. Clin Exp Metastasis, 32, 169-79.

Yui S, Nakamura T, Sato T, et al (2012). Functional engraftment of colon epithelium expanded in vitro from a single adult Lgr5 + stem cell. Nat Med, 18, 618-23.

Ziskin JL, Dunlap D, Yaylaoglu M, et al (2013). In situ validation of an intestinal stem cell signature in colorectal cancer. Gut, 62, 1012-23.

\section{(ब) $\odot \otimes$}

This work is licensed under a Creative Commons AttributionNon Commercial 4.0 International License. 